\title{
Immunohistochemical study of macrophage migration inhibitory factor in rat liver fibrosis induced by thioacetamide
}

\author{
Y. Hori, ${ }^{*}$ S. Sato, ${ }^{*}$ J. Yamate, ${ }^{\circ}$ M. Kurasaki, ${ }^{\sharp}$ J. Nishihira, ${ }^{\circledR}$ T. Hosokawa, ${ }^{+}$H. Fujita, ${ }^{\wedge}$ T. Saito^ \\ *Department of Food and Nutrition, Hakodate Junior College, Hakodate, Japan; ${ }^{\circ}$ Department of \\ Veterinary Pathology, Graduate School of Agriculture and Biological Sciences, Osaka Prefecture \\ University, Osaka; "Department of Environmental Medicine and Informatics, Graduate School of \\ Environmental Earth Science, Hokkaido University, Sapporo; 'Department of Molecular Chemistry, \\ Hokkaido University School of Medicine, Sapporo; 'Research Division for Higher Education, Center for \\ Research and Development in Higher Education, Hokkaido University, Sapporo; 'Laboratory of \\ Environmental Biology, Hokkaido University School of Medicine, Sapporo; Japan
}

(C)2003, European Journal of Histochemistry

Macrophage migration inhibitory factor (MIF) is a molecule known to regulate macrophage accumulation at sites of inflammation. To elucidate the role of MIF in progression of liver fibrosis, the immunohistochemical localization of MIF and macrophages in the liver were examined. Male Wistar rats received thioacetamide (TA) injections (200 mg/ kg, i.p.) for 1 or 6 weeks. In biochemical and histological tests, it was confirmed that liver fibrosis was induced. In immunohistochemical analyses, the expression of MIF protein was seen in hepatocytes in the areas extending out from the central veins to the portal tracts. In particular, at 6 weeks, immunoreactivity was detected in degenerated hepatocytes adjacent to the fibrotic areas but hardly observed in the fibrotic areas. On the other hand, a number of exudate macrophages stained by antibody ED1 were seen in the areas from the central veins to the portal tracts at 1 week and in the fibrotic areas at 6 weeks. Macrophages also showed a significant increase in number as compared with controls. These results revealed that there was a close relationship between the appearance of MIF expression and ED1-positive exudate macrophages in degenerated hepatocytes during the progression of TA-induced liver fibrosis.

Correspondence: Dr. Takeshi Saito, Laboratory of Environmental Biology, Hokkaido University School of Medicine, Sapporo 060-8638 Japan. Phone: international +81-11706-5065. Fax: international +81-11-706-7819

E-mail: taksaito@med.hokudai.ac.jp

Key words: macrophage migration inhibitory factor, macrophage, liver cirrhosis, thioacetamide, rat.

Paper accepted on June 4, 2003

European Journal of Histochemistry

2003; vol. 47 issue 4 [Oct-Dec]: 317-324 $\square$ ibrosis is known as a disorder evoked through a complex pathologic process after tissue injury. Its development requires the participation of inflammatory cells and development of myofibroblasts (Yamate et al. 1999; 2000). In fibrogenesis, macrophages play an important role after liver tissue damage because cytokines and growth factors such as interleukin-1 (IL-1), tumor necrosis factor- $\alpha(T N F-\alpha)$ and transforming growth factor$\beta$ (TGF- $\beta$ ) are produced (Khalil et al. 1989; Johnson et al. 1992; Leist et al. 1995; Schuppan et al. 1999). Macrophage infiltration has been examined immunohistochemically. EDI is a mouse monoclonal antibody to rat macrophages, labeling blood monocytes and exudate macrophages (Dijkstra et al. 1985; Damoiseaux et al. 1994). Thioacetamide (TA) has been used to induce hepatic lesions. Continuous administration to rats produces strands of collagen fibers and then obvious nodular fibrosis (Zimmermann et al. 1987; Sato et al. 2000). Noda et al. (1996) observed ED1-positive macrophages in injured areas in the TA-treated rat liver.

One of key molecules in macrophage accumulation at sites of inflammatory injury is macrophage migration inhibitory factor (MIF). MIF, which was found as a product of activated $T$ cells that inhibited the random migration of macrophages in vitro and promoted macrophage accumulation in vivo, is known to function in inflammatory and immune responses (Nishihira, 2000; Nishihira and Ogata, 2001). In liver disease, necrosis and inflammation in ethanol-fed rats are associated with increased expression of MIF (Nanji et al. 2001). Moreover, Kumagi et al. (2001) reported that MIF was expressed by hepatocytes and by the infiltrating cells in specimens from patients with alcoholic hep- 
atitis. These reports suggested that MIF was an important mediator of the inflammatory response in liver disease. Thus, it has been believed that MIF is a pivotal cytokine that plays an important role in inflammatory processes (Bacher et al. 1997; Lan et al. 2000). However, the relationship between MIF and infiltrating macrophages in the progression of liver fibrosis/cirrhosis is not adequately understood.

To gain insights into the possible role of MIF in the progression of fibrosis in the liver, we examined the immunohistochemical localization of MIF in rat liver fibrosis induced by repeated TA administrations. In addition, the relationship between MIF production in hepatocytes and macrophages infiltrating in the areas of fibrosis is discussed.

\section{Materials and Methods}

\section{Animals and TA administration}

Male Wistar rats (Charles River Japan, Shiga, Japan) with a starting weight of 161-181 g were used. They were maintained under 12 -h dark-light cycle and with CE-2 standard pellets (Clea Japan Inc., Tokyo, Japan) and tap water ad libitum. The animals were divided into two groups: control rats $(n=8)$ received i.p. injections of saline and the other group of rats $(n=8)$ were injected i.p. with 200 $\mathrm{mg} / \mathrm{kg}$ of TA, three times a week for 1 or 6 weeks. At 3 days after the final TA injection, the animals were used for experiments. All procedures were performed according to the regulations in Guide for the Care and Use of Laboratory Animals as defined by the NIH.

\section{Sample collection}

Before the animals were killed after the final injection, blood samples were collected under ether anesthesia and livers were quickly removed, rapidly rinsed, weighed and fixed in 10\% formalin solution. Portions of each liver were stored at $-80^{\circ} \mathrm{C}$ until use.

\section{Biochemical assays}

Plasma samples were obtained after centrifugation (3,000 rpm for $10 \mathrm{~min})$ and were tested for aspartate aminotransferase (AST), alanine aminotransferase (ALT) and alkaline phosphatase (ALP) according to the instruction manuals of diagnostic reagent kits of Wako Pure Chemical Industries, Ltd. (Osaka, Japan).

\section{Histopathology}

At sacrifice, body and liver weights were measured. For histologic examination, the formalin-fixed kidney tissues were embedded in paraffin, and sections $6 \mu \mathrm{m}$ in thickness were stained with hematoxylin-eosin (HE) and by Azan stain for collagen fibers.

\section{Immunohistochemistry}

The avidin-biotin complex method (LSAB 2kit; Dako Corp., Carpinteria, CA, USA) was employed using deparaffinized sections according to the method of Yamate et al. (2000) with minor modifications. Antibody EDI was obtained from Chemicon International, Inc. (Temecula, USA). A polyclonal anti-rat MIF antibody was generated by immunizing New Zealand white rabbits with purified recombinant rat MIF according to the methods described by Nishihira et al. (1995). The specificity of the MIF antibody was confirmed as described previously (Nishihira et al. 1995). Briefly, for EDl and MIF expression, deparaffinized sections were pretreated with $0.1 \%$ trypsin solution in phosphate-buffered saline (PBS), pH 7.4, for 15 min at $37^{\circ} \mathrm{C}$ and $3 \% \mathrm{H} 202$ for 10 min at room temperature. Sections were then preincubated with $1.5 \%$ skimmed milk in PBS for 30 min at room temperature, and incubated for $14 \mathrm{~h}$ at $4^{\circ} \mathrm{C}$ with the antirat MIF antibody (1:500) or EDI antibody (1:200), which were diluted in PBS including $1 \%$ bovine serum albumin. Thereafter, they were incubated with biotinylated anti-rabbit and anti-mouse immunoglobulins for 10 min. Final incubation was carried out for 10 min with streptavidin conjugated to horseradish peroxidase, and positive reactions were visualized with 3,3'-diaminobenzidine tetrahydrochloride (DAB) in $50 \mathrm{mM}$ Tris- $\mathrm{HCl}$ buffer, $\mathrm{pH}$ 7.4 , containing $3 \% \mathrm{H}_{2} \mathrm{O}_{2}$. Sections were counterstained lightly with hematoxylin. Cells showing a distinct immunoreaction for EDI were counted in 5 randomly selected areas $\left(0.0625 \mathrm{~mm}^{2}\right.$ each) of sections at a magnification of x400 (Yamate et al. 1993)

\section{Statistical analysis}

Each value is expressed as mean \pm SEM. Statistical analyses were performed by one-way analysis of variance (ANOVA), followed by the Tukey test. 
Table 1. Body weight (BW) at autopsy, relative liver weight (RLW), and values of AST, ALT and ALP in control and TA-treated rats.

\begin{tabular}{|c|c|c|c|c|}
\hline & \multicolumn{2}{|c|}{1 week } & \multicolumn{2}{|c|}{6 weeks } \\
\hline & Control & TA-treated & Control & TA-treated \\
\hline BW (g) & $237.5 \pm 5.0$ & $194.3 \pm 2.4^{*}$ & $424.8 \pm 6.4$ & $302.8 \pm 14.0^{*}$ \\
\hline RLW (\%) & $4.9 \pm 0.1$ & $5.5 \pm 0.1^{*}$ & $4.6 \pm 0.2$ & $6.0 \pm 0.2^{*}$ \\
\hline AST (IU/I) & $23 \pm 1$ & $39 \pm 5^{*}$ & $26 \pm 0$ & $53 \pm 6^{*}$ \\
\hline ALT (IU/I) & $18 \pm 2$ & $47 \pm 17$ & $20 \pm 3$ & $23 \pm 2$ \\
\hline $\operatorname{ALP}(I U / I)$ & $208.5 \pm 7.8$ & $290.3 \pm 33.8$ & $157.9 \pm 9.4$ & $348.0 \pm 56.2^{*}$ \\
\hline
\end{tabular}

Values are expressed as mean \pm SEM $(n=4)$. *; $p<0.05$ compared with age-matched control.

\section{Results}

\section{TA-induced liver fibrosis}

As shown in Table 1, the body weight in TA-treated rats was significantly decreased when compared with control rats. The relative liver weight was increased in TA-treated rats. In biochemical tests, the levels of ALP and AST in TA-treated rats at 6 weeks were significantly elevated. TA injections for 6 weeks produced a roughened surface on the liver in every rat examined. Histologically, degenerating hepatocytes with eosinophilic, swollen cytoplasm were mainly observed in perivenular areas and occasionally in periportal areas. These hepatocytes often had hypertrophic nuclei at 6 weeks. By Azan staining, slight fibrosis was seen around the perivenular zone (Figure 1B). At 6 weeks, disarrangement of hepatic lobules, collagen fibers and stained fibrous septa were observed. There were also increased numbers of cells with a fibroblastlike morphology in the fibrotic areas (Figure 1C). No noticeable changes were seen in controls (Figure 1A). Our biochemical and histological findings indicated that the liver fibrosis in rats was induced by repeated i.p. injections of TA.

\section{Localization of MIF protein and ED1-positive macrophages}

To examine the relationships between the appearance of ED1-positive exudate macrophages and MIF expression in the liver fibrosis of rats, we carried out immunohistochemical analyses. The localization of MIF protein in the liver in TA-injected rats is shown in Figure $2 \mathrm{~A}, \mathrm{C}$ and $\mathrm{E}$. Immunoreactivity against the MIF antibody in the parenchymal cells, especially in the hepatocytes at 1 week, was seen linearly from the central veins to portal tracts (Figure 2C). At 6 weeks, stronger immunoreactivity was observed in the degenerated
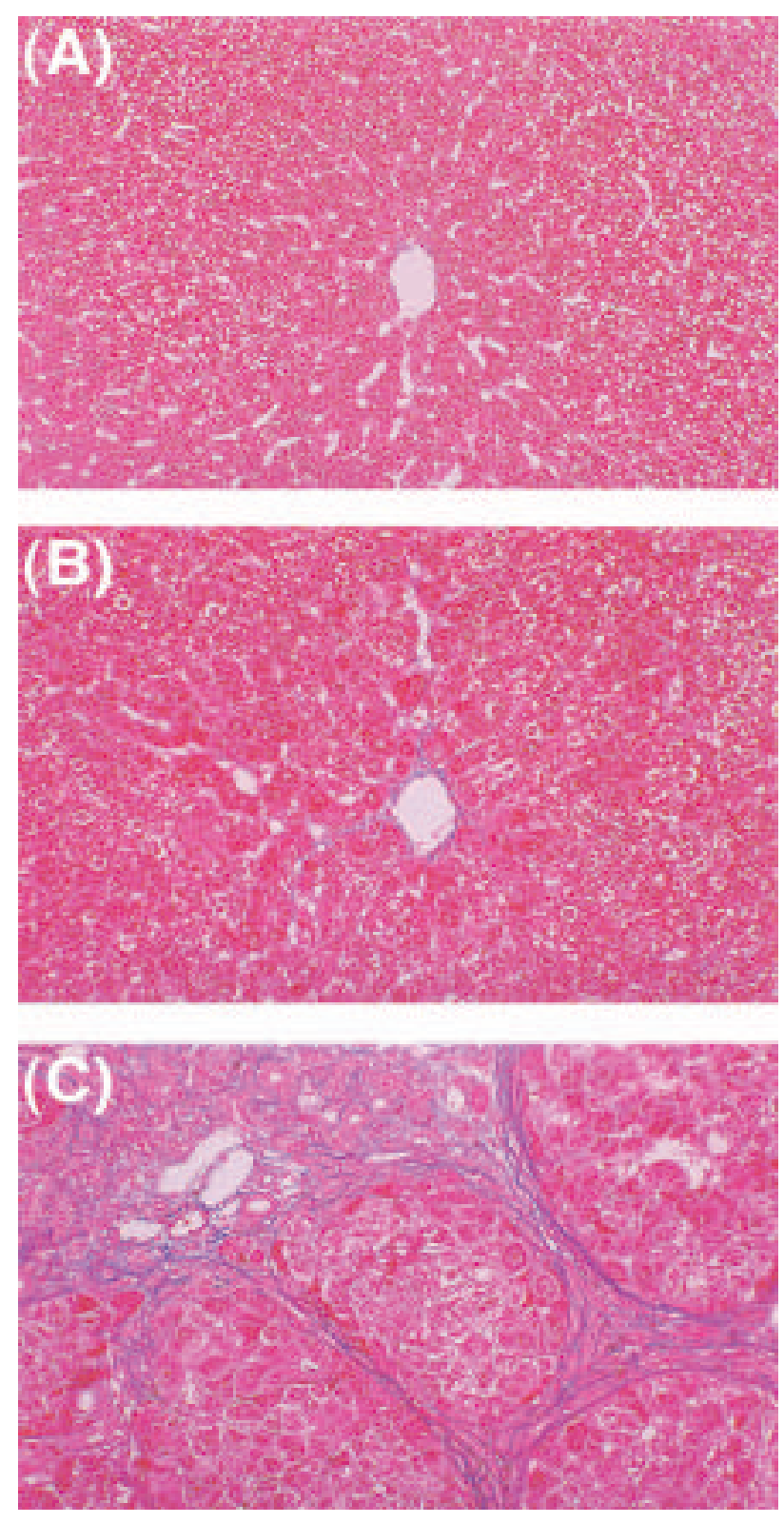

Figure 1. Light micrographs of rat livers. Collagen stain with Azan. A: liver from a normal rat. B: liver from a rat treated with TA for 1 week, showing slight fibrosis around the perivenular zone. C: liver from a rat treated with TA for 6 weeks, showing distinct fibrous septa surrounding nodules. Original magnification x 60 . 

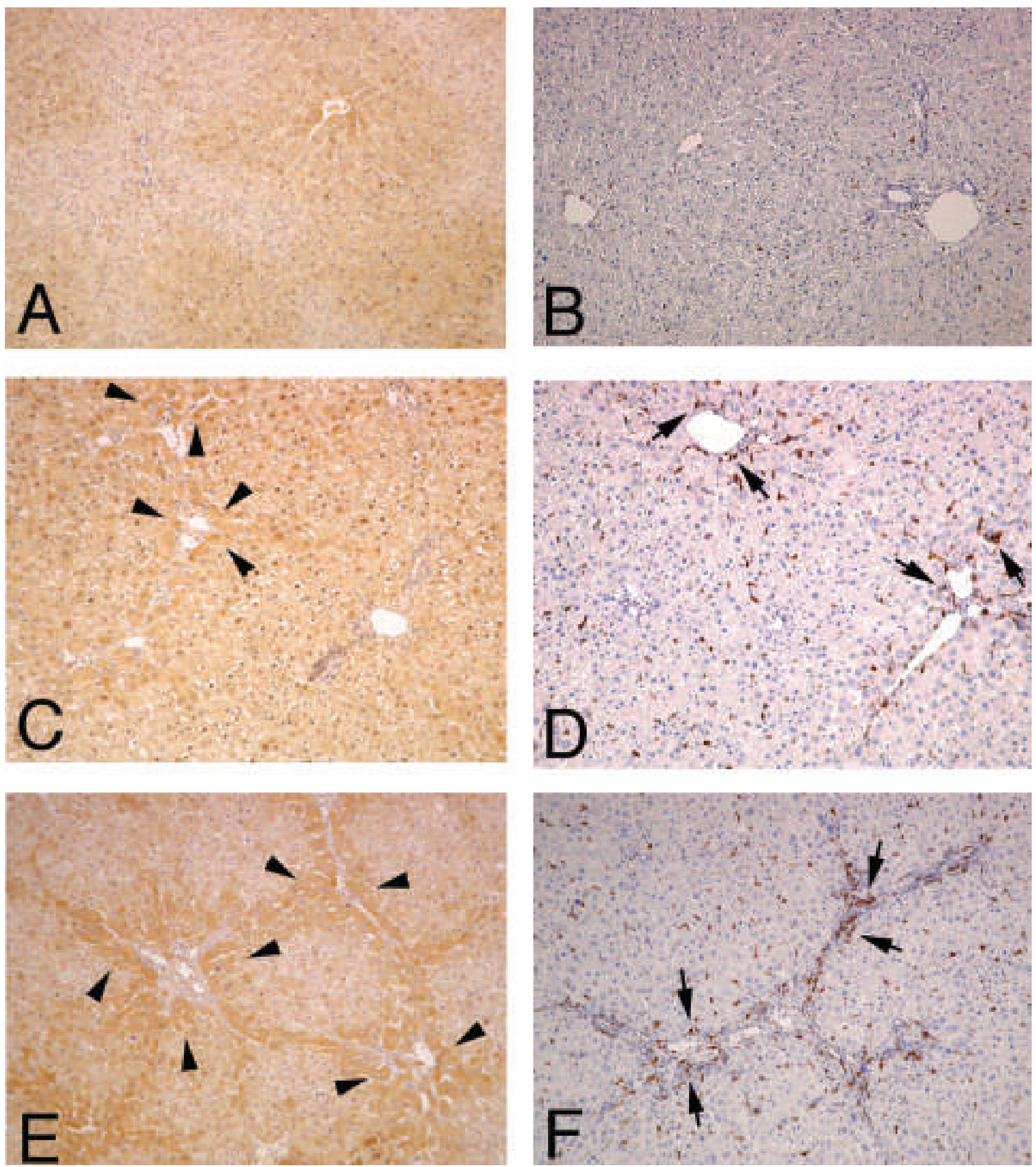

Figure 2. Immunohistochemical localization of MIF protein (A, C, E) and ED1-positive macrophages (B, D, F) in rat livers. Immunoreactivity against the MIF antibody in the control (A) is weaker than those of 1 week (C) and 6 weeks (E). At 6 weeks, stronger staining was present in the parenchymal cells adjacent to the areas of fibrosis. The stronger staining against the MIF antibody is shown as arrowheads in C and E. ED1-positive macrophages are distributed in perivenular and periportal areas at 1 week (D), and in fibrotic areas at 6 weeks (F), increasing in number when compared with controls (B). ED1-positive macrophages are shown as arrows in $D$ and $F$. Counterstained with hematoxylin. Original magnification x 60. 

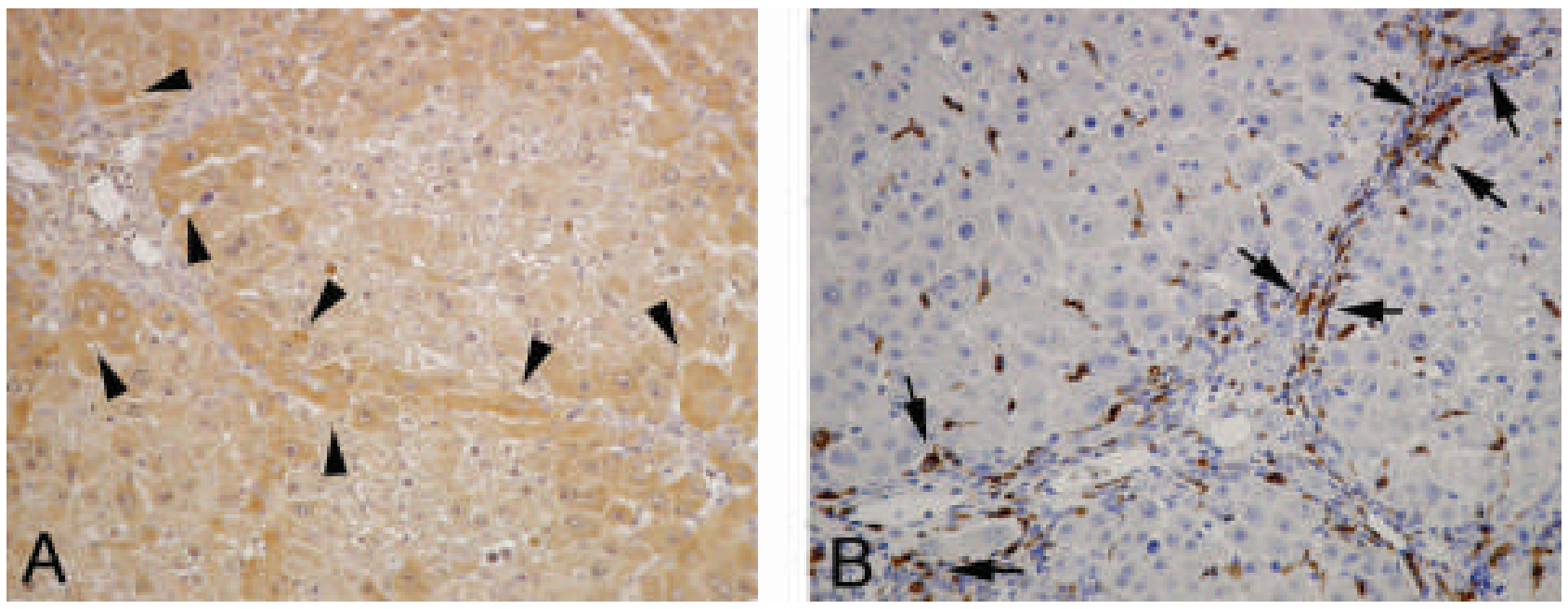

Figure 3. Higher magnification view of the localization of MIF protein and ED1-positive macrophages in liver from a rat treated with TA for 6 weeks. A: immunoreactivity against the MIF antibody (arrowheads) is observed in the degenerated hepatocytes, present around the fibrotic areas. B: a large number of ED1-positive macrophages (arrows) are seen in the fibrotic areas. Original magnification $x 300$.

hepatocytes present around the fibrotic areas (Figure 2E). In controls, MIF immunoreactivity was weakly detectable in the liver, and relatively strong staining was seen in the hepatocytes surrounding the central veins (Figure $2 \mathrm{~A}$ ). These findings indicated that the expression of MIF was mainly activated in parenchymal cells, especially in the hepatocytes of rats with progressive liver fibrosis.

The localization of ED1-positive macrophages in the liver in TA-injected rats is shown in Figure 2B, $D$ and F. EDl-positive macrophages emerged in perivenular and periportal areas where hepatocyte degeneration and subsequent collagen deposition occurred at 1 week (Figure 2D). A large number of ED1-positive macrophages were seen mainly in the fibrotic areas and along the sinusoidal walls in TAtreated rats at 6 weeks (Figure 2F). In controls, a few EDl-positive macrophages were observed in the areas surrounding the central veins (Figure $2 \mathrm{~B}$ ). These results show the infiltration and accumulation of macrophages in the progressive liver fibrosis of rats. Interestingly, stronger immunoreactivity against the MIF antibody was observed in the degenerated hepatocytes present around the fibrotic areas at 6 weeks (Figure 3A), whereas the signals were hardly seen in fibrotic areas in which a large number of ED1-positive macrophages infiltrated (Figure 3B).

To determine the kinetics of macrophages in the TA-treated rat liver, we measured the number of ED1-positive macrophages (Table 2). Compared with control rats, ED1-positive macrophages in TAtreated rats showed significant increases in number at both 1 and 6 weeks $(P<0.001)$. However, there was no significant difference in the numbers in TAtreated rats between 1 and 6 weeks.

\section{Discussion}

In this study, we investigated the immunohistochemical localization of MIF protein and macrophages in the progression of liver fibrosis. The main

Table 2. Number of ED1-positive macrophages (cells/0.0625 $\mu \mathrm{m}^{2}$ ) in livers of control and TA-treated rats.

\begin{tabular}{|c|c|c|c|c|}
\hline & \multicolumn{2}{|c|}{1 week } & \multicolumn{2}{|c|}{6 weeks } \\
\hline & Control & TA-treated & Control & TA-treated \\
\hline Number of ED1-positive macrophages & $7.1 \pm 0.5$ & $45.5 \pm 1.6^{* *}$ & $6.3 \pm 0.6$ & $46.7 \pm 2.5^{* *}$ \\
\hline
\end{tabular}

Values are expressed as mean \pm SEM $(n=4) .{ }^{* *} ; P<0.001$ compared with age-matched control. 
findings of this study were strong expression of MIF in the parenchymal areas and a great increase in number of ED1-positive macrophages in the fibrotic areas in the livers of rats induced by repeated TA injections. On the other hand, expression of MIF was hardly observed in the fibrotic areas. It was reported that MIF staining was observed in hepatocytes of liver specimens from patients with liver cirrhosis (Akbar et al. 2001). Kumagi et al. (2001) described an increase of MIF levels in serum and localization of MIF-expressing cells in the liver in patients with alcoholic hepatitis. On the other hand, it was reported that macrophages might act as an early trigger in the genesis of hepatic fibrosis (Noda et al. 1996; Yamate et al. 1999). However, to the best of our knowledge, the relationship between MIF expression and infiltrating macrophages in the progression of liver fibrosis/cirrhosis has not been adequately discussed.

TA-induced hepatic fibrosis/cirrhosis in rats is histologically similar to human cirrhosis (Zimmermann et al. 1987). Our histological examination confirmed degenerating hepatocytes at 1 week, and collagen fibrils and obvious nodular fibrosis at 6 weeks (Figure $1 \mathrm{~B}$ and $\mathrm{C}$ ). These findings were supported by previous reports that continuous administration of $0.03 \%$ TA in drinking water or repeated i.p. injections into rats produced micronodular cirrhosis (Zimmermann et al. 1987; Mori et al. 1993; Sato et al. 2000). In addition, the levels of ALP and AST in TA-treated rats increased significantly compared with the control, as previously reported (Fontana et al. 1996).

In this study, a number of ED1-positive macrophages were seen in the areas from the central veins to the portal tracts at 1 week (Figure 2D). At 6 weeks, a similar number of macrophages and enlargement in cell size were found in the areas of fibrosis (Figure 2F). Noda et al. (1996) reported that changes in the hepatic macrophage population were maintained during 12 weeks of TA administration. These findings indicated that macrophages were quickly recruited at the early stage of the liver fibrosis. Thus, it is likely that macrophages play an important role in the pathogenesis of liver fibrosis (Noda et al. 1996; Yamate et al. 1999).

It is interesting that, in this study, increased expression of MIF was present in the parenchymal areas adjacent to the areas of fibrosis at 6 weeks (Figure 2E). Even at lweek, the expression was seen in the hepatocytes that formed a line spread out from the central veins to the portal tracts (Figure 2C). On the other hand, immunoreactivity against the MIF antibody was hardly observed in the fibrotic areas, whereas macrophages stained by the EDI antibody were present. The source of MIF production has been shown to be the monocyte/macrophage (Leech et al. 1999; Nishihira, 2000), while glomerular and tubular endothelial cells, and fibroblast-like synoviocytes also contribute to MIF expression (Lan et al. 1997; Leech et al. 1999). Previous reports indicated that the up-regulation of MIF in hepatocytes was associated with inflammatory changes in the liver in ethanol-fed and lipopolysaccharide-induced rats (Bacher et al. 1997; Nanji et al.2001). Therefore, we suggest that, in the progressive liver fibrosis induced by $\mathrm{TA}$, the main source of MIF production might be the hepatocytes rather than macrophages in the fibrotic areas.

The present study demonstrated that there was a close relationship between the appearance of ED1positive exudate macrophages and MIF expression in degenerated hepatocytes in the progression of TA-induced liver fibrosis, suggesting that MIF produced by the degenerated hepatocytes might be related to halt the migration of EDI-positve macrophages and promote their accumulation in fibrotic areas. The fibrotic areas are formed by myofibroblastic cells and the extracellular matrixes they produce (Friedman, 1999; Schuppan et al. 1999). The ED1-positive macrophages observed in the fibrotic areas, in which infiltration might be affected with MIF produced in degenerated hepatocytes, may be associated with the development of myofibroblast-like cells, at least in part, because such macrophages can produce TNF- $\alpha$ and TGF- $\beta$ (Khalil et al. 1989; Leist et al. 1995). It has been reported that Kupffer cells, resident macrophages, are considered to be responsible for progression of hepatic fibrosis (Luckey and Petersen, 2001; Melgert et al. 2001). Thus, the association of resident macrophages with MIF expression should be investigated in further studies.

\section{Acknowledgments}

We wish to thank Ms. Yoko Shimizu of Hakodate Junior College for her technical assistance during our work. This work was supported in part by Grants-in-Aid from the Ministry of Education, Culture, Sports, Science and Technology in Japan. 


\section{References}

Akbar SM, Abe M, Murakami H, Tanimoto K, Kumagi T, Yamashita $Y$, et al. Macrophage migration inhibitory factor in hepatocellular carcinoma and liver cirrhosis; relevance to pathogenesis. Cancer Lett 2001;171:125-32.

Bacher M, Meinhardt A, Lan HY, Mu W, Metz CN, Chesney JA, et al. Migration inhibitory factor expression in experimentally induced endotoxemia. Am J Pathol 1997;150:235-46.

Damoiseaux JG, Dopp EA, Calame W, Chao D, Macpherson GG, Dijkstra CD. Rat macrophage lysosomal membrane antigen recognized by monoclonal antibody ED1. Immunology 1994;83:140-7.

Dijkstra CD, Dopp EA, Joling P, Kraal G. The heterogeneity of mononuclear phagocytes in lymphoid organs: distinct macrophage subpopulations in the rat recognized by monoclonal antibodies ED1, ED2 and ED3. Immunology 1985;54:589-99.

Fontana L, Moreira E, Torres MI, Fernandez MI, Rios A, Sanchez de Medina $F$, et al. Serum amino acid changes in rats with thioacetamide-induced liver cirrhosis. Toxicology 1996;106:197-206.

Friedman SL. Cytokines and fibrogenesis. Semin Liver Dis 1999;19: 129-40.

Hellerbrand C, Stefanovic B, Giordano F, Burchardt ER, Brenner DA. The role of TGF-betal in initiating hepatic stellate cell activation in vivo. J Hepatol 1999;30:77-87.

Johnson SJ, Hines JE, Burt AD. Macrophage and perisinusoidal cell kinetics in acute liver injury. J Pathol 1992;166:351-58.

Khalil N, Bereznay 0, Sporn MB, Greenberg AH. Macrophage production of transforming growth factor $\dot{E}_{i}$ and fibroblast collagen synthesis in chronic pulmonary inflammation. J Exp Med 1989;170: 727-37.

Kumagi T, Akbar F, Horiike N, Onji M. Increased serum levels of macrophage migration inhibitory factor in alcoholic liver diseases and their expression in liver tissues. Clin Biochem 2001;34:189-93.

Lan HY, Bacher M, Yang N, Mu W, Nikolic-Paterson Dj, Metz C, et al. The pathogenic role of macrophage migration inhibitory factor in immunologically induced kidney disease in the rat. J Exp Med 1997. 185:1455-65.

Lan HY, Yang N, Nikolic-Paterson DJ, Yu XQ, Mu W, Isbel NM, et al. Expression of macrophage migration inhibitory factor in human glomerulonephritis. Kidney Int 2000;57:499-509.

Leech M, Metz C, Hall P, Hutchinson P, Gianis K, Smith M, et al. Macrophage migration inhibitory factor in rheumatoid arthritis: evidence of proinflammatory function and regulation by glucocorticoids. Arthritis Rheum 1999;42:1601-8.

Leist M, Gantner F, Bohlinger I, Tiegs G, Germann PG, Wendel A. Tumor necrosis factor-induced hepatocyte apoptosis precedes liver failure in experimental murine shock models. Am J Pathol 1995: 146:1220-34.
Luckey SW, Petersen DR. Activation of Kupffer cells during the course of carbon tetrachloride-induced liver injury and fibrosis in rats. Exp Mol Pathol 2001;71:226-40.

Melgert BN, Olinga P, Van Der Laan J M, Weert B, Cho J, Schuppan D, et al. Targeting dexamethasone to Kupffer cells: effects on liver inflammation and fibrosis in rats. Hepatology 2001;34:719-28.

Mori T, Okanoue T, Sawa Y, Hori N, Ohta M, Kagawa K. Defenestration of the sinusoidal endothelial cell in a rat model of cirrhosis. Hepatology 1993;17:891-7.

Nanji AA, Lau GKK, Tipoe GL, Yuen ST, Chen YX, Thomas P, et al. Macrophage migration inhibitory factor expression in male and female ethanol-fed rats. J Interferon Cytokine Res 2001;21:105562.

Nishihira J, Kuriyama T, Sakai M, Nishi S, Ohki S, Hikichi K. The structure and physicochemical properties of rat liver macrophage migration inhibitory factor. Biochim Biophys Acta 1995;1247:159-62.

Nishihira J. Macrophage migration inhibitory factor (MIF): Its essential role in the immune system and cell growth. J Interferon Cytokine Res 2000;20:751-62.

Nishihira J, Ogata A. Macrophage migration inhibitory factor as a target molecule in multiple sclerosis. Curr Opin Investig Drugs 2001;2: 778-82.

Noda S, Masumi S, Moriyama M, Kannan Y, Ohta M, Sugano T, et al. Population of hepatic macrophages and response of perfused liver to platelet-activating factor during production of thioacetamideinduced cirrhosis in rats. Hepatology 1996;24:412-8.

Sato M, Kakubari M, Kawamura M, Sugimoto J, Matsumoto K, Ishii T. The decrease in total collagen fibers in the liver by hepatocyte growth factor after formation of cirrhosis induced by thioacetamide. Biochem Pharmacol 2000;59:681-90.

Sato S, Shimizu M, Hosokawa T, Saito T, Okabe M, Niioka T, et al. Distribution of zinc-binding metallothionein in cirrhotic liver of rats administered zinc. Pharmacol Toxicol 2000;87:292-6.

Schuppan D, Cho JJ, Jia JD, Hahn EG. Interplay of matrix and myofibroblasts during hepatic fibrogenesis. Curr Top Pathol 1999;93: 205-18.

Yamate J, Kumagai D, Tsujino K, Nakatsuji S, Kuwamura M, Kotani T, et al. Macrophage populations and apoptotic cells in the liver before spontaneous hepatitis in Long-Evans Cinnamon (LEC) rats. J Com Pathol 1999;120:333-46.

Yamate J, Sato K, Machida Y, Ide M, Sato S, Nakatsuji S, et al. Cisplatin-induced rat renal interstitial fibrosis; a possible pathogenesis based on the data. J Toxicol Pathol 2000;13:237-47.

Yamate J, Tatsumi M, Nakatsuji S, Kuwamura M, Kotani T, Sakuma S. Immunohistochemical observations of macrophages and perisinusoidal cells in carbon tetrachloride-induced rat liver injury. J Vet Med Sci 1993;55:973-7.

Zimmermann T, Müller A, Machnik G, Franke $H$, Schubert $H$, Dargel R. Biochemical and morphological studies on production and regression of experimental liver cirrhosis induced by thioacetamide in Uje:WIST rats. Z Versuchstierkd 1987;30:165-80. 
Y. Hori et al. 